\title{
NヨSIE: a fiber-fed near-infrared spectrograph for TIGRE telescope
}

\section{Kintziger, G. Rauw, R. Desselle, P. Rochus, J. Loicq}

C. Kintziger, G. Rauw, R. Desselle, P. Rochus, J. Loicq, "NヨSIE: a fiber-fed near-infrared spectrograph for TIGRE telescope," Proc. SPIE 10702, Groundbased and Airborne Instrumentation for Astronomy VII, 107024Y (6 July 2018); doi: $10.1117 / 12.2312586$

Event: SPIE Astronomical Telescopes + Instrumentation, 2018, Austin, Texas, United States 


\title{
NGSIE: a fiber-fed near-infrared spectrograph for TIGRE telescope
}

\author{
C. Kintziger*a, G. Rauw ${ }^{\mathrm{b}}$, R. Desselle ${ }^{\mathrm{a}}$, P. Rochus ${ }^{\mathrm{a}}$, Jérôme Loicq ${ }^{\mathrm{a}}$ \\ ${ }^{a}$ Centre Spatial de Liege, Avenue du Pré-Aily, 4030 Angleur, Belgium; ${ }^{b}$ Groupe d'Astrophysique \\ des Hautes Energies, Institut d'Astrophysique et de Géophysique, Université de Liège, Allée du 6 \\ Août, 19c, Bât B5c, 4000 Liège, Belgium
}

\begin{abstract}
Our contribution intends to present the obtained performances of the NGSIE instrument, a new near-infrared fiber-fed spectrograph developed at the University of Liège. This instrument was developed, aligned and tested at the Centre Spatial de Liège and first light was achieved in October 2017. This paper will go through the alignment process and optical performance verification to eventually introduce the first light observations. The final location of NGSIE will be the TIGRE telescope located in La Luz, Mexico. The observational data provided by this instrument will help several research groups from the University of Liège to study massive stars. In particularly, evolution models will be improved through the comparison of the collected spectra with theoretical models. This collaboration will therefore contribute to a better understanding of massive stars and the mechanisms that take place within these extraordinary objects.
\end{abstract}

Keywords: Near-infrared spectroscopy, instrumentation, massive stars, TIGRE telescope, alignment, ground-based observation

\section{INTRODUCTION}

\subsection{Research project}

The global research project that involves the present instrument development aims at studying massive stars. This ARC (Action de Recherche Concertée) gathers three separate research groups from the University of Liège (ULiège): the Groupe d'Astrophysique des Hautes Energies (GAPHE), the Astrophysique Stellaire Théorique et Astérosismologie (ASTA) and the Centre Spatial de Liège (CSL). The GAPHE research group is in charge of the observation of massive stars at different wavelengths. These involve the TIGRE ${ }^{1}$ telescope that will host the spectrographic instrument presented throughout this paper. The ASTA scientific unit on the other hand focuses on theoretical simulations which aim at studying the interior of massive stars and explain their evolution process. To do so, they use among other things the asteroseismology technique. The CSL eventually covers technical aspects and within this ARC, CSL is in charge of the conception of innovative scientific instruments to study massive stars.

The main question to which the research project intends to bring some enlightening answer elements is: how do massive stars evolve? Part of the answer may reside in the mutual influences that exist between the members of binary systems. The stellar rotation must also be considered to properly assess the induced effects on the evolution processes. The existence of a magnetic field and the mass loss experienced by such stars also need to be included in the stellar evolution models. The research project therefore intends to assess all these aspects by improving the associated models and collect useful data with the developed instruments. CSL is therefore in charge of the development of new dedicated instruments to provide scientific data to the other research units. These will be used to investigate the above hypotheses and improve the numerical models. A better understanding of massive stars will therefore result from this interaction between the groups involved in the ARC.

Today's instrumentation is largely dominated by oversubscribed telescopes which focus on a limited number of "fashionable" research topics ${ }^{2}$. The consequence is a lack of time for the study of massive stars, especially for long campaigns. The general actual opinion usually considers that large telescopes are better suited for spectroscopic astronomical purposes. This idea is correct when considering deep-sky observations that induce low-contrast imaging. For example, the observation of Quasars and Galaxies with small telescopes usually leads to low signal-to-noise ratio spectra and these small apertures do not actively participate to this research field ${ }^{2}$.

*ckintziger@uliege.be; phone +3243824679

Ground-based and Airborne Instrumentation for Astronomy VII, edited by Christopher J. Evans, Luc Simard, Hideki Takami, Proc. of SPIE Vol. 10702, 107024Y · ( 2018 SPIE · CCC code: 0277-786X/18/\$18 · doi: 10.1117/12.2312586 
The study of massive stars does not obey to this rule. Small telescopes can be used in association to low-cost instruments to conduct scientific research on stellar physics. The monitoring of the varying spectra of bright emission lines can for example be carried out with such observatories. Smaller telescopes are also naturally more numerous and easier to access than large observational platforms. This advantage therefore benefits to long-duration spectroscopic campaigns which can support other observations by larger platforms that focus on the determination of detailed spectroscopic parameters ${ }^{2}$. This interesting niche can therefore bring the light on poorly understood properties of massive stars and the present project intends to position our research group association as a key player in this field. This partnership intends to gather the individual expertise and give birth to a new instrument. This spectrograph will first be used on the TIGRE telescope that can be accessed by the University of Liège and is located in Mexico.

\subsection{Massive stars}

Massive stars exhibit large masses and extreme luminosities. These objects are usually the first ones we observe when targeting at galaxies ${ }^{2}$. However, these stars are also very rare: for one typical massive star in the Milky Way, there exist approximately a hundred thousand solar-type stars ${ }^{3}$. The enormous distance that separates us from the closest objects requires specific observing techniques. Indeed, standard imaging methods and even interferometric observations from combined telescopes are insufficient to provide enough resolving power. Most distance objects are therefore rather studied through spectroscopic analyses which are the key for understanding the $\operatorname{cosmos}^{2}$. The visibility of massive stars from large distances also enables probing the conditions of both their own environment and the intermediate space that separates us from those objects ${ }^{4}$. The observation of those rare objects may however be also problematic. Their visibility may indeed be threatened at early formation phases due to the surrounding dust. Some important evolution stages are also short in time and these objects usually appear in groups which complicates the understanding of evolution processes due to mutual interactions 5 .

The spectroscopic analysis of bright massive stars does not require large telescopes as previously inferred. Indeed, the study of line profiles with the help of spectrographs can be performed with small telescopes when targeting massive stars. Moreover, the typical accessible time-scales for those objects range from a few minutes to several years. Their intense brightness also usually enables their observation from polluted areas close to urban centers. This benefits to amateur astronomy that can develop and supply useful complementary data to professional scientists. Long-term campaigns are usually also conducted with smaller telescopes as they are easier to access than oversubscribed large platforms.

Stars must exhibit an initial mass of approximately 8 solar masses to be qualified as massive ${ }^{4}$. These "cosmic engines" highly influence their environment. Indeed, during the major part of their life, they pour to the interstellar medium high quantities of ionizing photons. On the other hand, intense and fast stellar winds are generated due to their high luminosity and irradiate their surrounding medium. Massive stars are the major source of both ultraviolet ionizing radiation in galaxies and infrared-luminosity which originates from heated dust ${ }^{3}$. These stars also represent the major manufacturers of carbon, nitrogen and oxygen and enrich the interstellar medium. The turbulence induced by these exchanges with their environment strongly affects the formation of stars and planets and the structure of galaxies ${ }^{5}$.

Their death generally occurs as a gigantic supernova. This final explosion ejects several components into the neighborhood. This violent event is indeed the source of the production of newly synthesized chemical elements ${ }^{4}$. The black hole formation that may follow when the star collapses is the source of gamma-ray bursts which is believed by many to be the most energetic phenomenon yet found ${ }^{3}$.

The construction of accurate models of the evolution of massive stars is therefore of high importance to properly understand all these mechanisms. Internal mixing, mass loss and binarity are three elements that still need more accurate models ${ }^{4}$. This accomplishment cannot be achieved without the collection of observational data to compare with theoretical information. Indeed, defects of the models can only be highlighted by observations which in turn are used to improve the numerical simulations ${ }^{3}$.

The ARC partnership therefore intends to shed new light onto these poorly understood processes. Modeling improvements are conducted by trying to include mechanisms such as stellar rotation, mass loss, magnetic field and binarity. On the other hand, new instrumentation is under study or development by the CSL to provide observational data and compare the results with theoretical predictions. 


\subsection{Proposed spectrograph instrument project}

The scientific motivation which drove the conception of the NGSIE spectrograph was filling an existing gap in groundbased spectroscopic instrumentation. Indeed, even though this waveband is accessible from the ground, the near-infrared window around 1-1.1 $\mu \mathrm{m}$ is rather neglected. The lack in efficiency of typical CCDs is probably the major reason why such observations are not conducted. However, many spectral lines are located within this spectral interval and can be used to diagnose stellar activity and stellar winds, He I $\lambda 10830$ is an example. Once delivered to its final location at TIGRE, the instrument will study this faint region of the spectrum and provide data to try to unveil some misunderstood phenomenon's taking place in massive stars.

The development of the instrument ended to a Czerny-Turner spectrograph within which a toroidal lens has been inserted to deal with astigmatism. The optical requirements for this instrument are summarized in Table 1 below.

Table 1. Scientific requirements on the near-infrared spectrograph

\begin{tabular}{|l|l|l|}
\hline \multicolumn{1}{|c|}{ Parameter } & \multicolumn{1}{c|}{ Requirement } & \multicolumn{1}{c|}{ Goal } \\
\hline Spectral range & $1000-1100 \mathrm{~nm}$ & $940-1400 \mathrm{~nm}$ \\
\hline Resolving power & 10000 & 20000 \\
\hline Target magnitudes & $\mathrm{V}<7$ & $\mathrm{~V}<9$ \\
\hline $\begin{array}{l}\text { Signal-to-noise ratio in } \\
\text { continuum }\end{array}$ & 100 at $\mathrm{V}=6$ & 100 at $\mathrm{V}=7$ \\
\hline Typical exposure time & $15-30 \mathrm{~min}$ & $15 \mathrm{~min}$ \\
\hline $\begin{array}{l}\text { Simultaneous sky } \\
\text { measurements yes }\end{array}$ & yes \\
\hline $\begin{array}{l}\text { Wavelength calibration } \\
\text { accuracy }\end{array}$ & $\delta \lambda / 20=0.05 \AA$ & $\delta \lambda / 20=0.025 \AA$ \\
\hline
\end{tabular}

The purpose of this contribution is to introduce the optical performances of the instrument obtained after alignment within CSL installation as well as the first observations from Liege. The optical design and analyses of NGSIE have already been deeply covered in previous contributions and will not be discussed within this paper. For further explanations on the conception steps, the author suggests referring to the relevant references ${ }^{6,7}$.

\subsection{Bundle configuration}

A bundle a 15 fibers is selected to link the telescope to the spectrograph, located in a separate room beside the dome. Figure 1 introduces the selected fiber configuration at each side of the bundle. The star light focused at the telescope focal plane is collected with the help of fibers located at the centre of the circular-shaped bundle input. These latter are set to form a square of 3 by 3 elements, which is reminiscent of the configuration Barden adopted at Kitt Peak National Observatory (KPNO) ${ }^{8}$. The great advantage of using a fiber bundle over masks to perform multi-object spectroscopy is the total freedom of fiber arrangement at both telescope and spectrograph sides ${ }^{9}$. The selected arrangement for our scientific case is explained below.

On the other hand, 6 other "satellite" fibers surround the "star" ones in order to sample the sky background. This way, the sky spectrum is recorded aside of the star signal and can thereafter be subtracted to obtain accurate target spectra. The satellite fibers lie on a circle of diameter $1 \mathrm{~mm}$ centered onto the star fibers. This $500 \mu \mathrm{m}$ radius approximately corresponds to 10 arcsec on the sky when considering the TIGRE telescope, which ensures measuring the sky background far enough from the star to avoid contamination by star light and close enough to collect representative measurements. On the other hand, this fiber distribution may allow for sky background gradients to be eliminated without performing any chopping or nodding.

It must be noted that sky background measurement and subtraction techniques are very important as noted by Parry ${ }^{9}$. In contrast with slit spectrographs, fiber-fed instruments must develop accurate subtraction methodologies because the sky 
signal is usually sampled some distance away from the source by a dedicated fiber. Moreover, the related spectrum is also imaged on a separate portion of the detector. Therefore, systematic errors may occur due to scattering or vignetting in the spectrograph for example?

At the spectrograph's side, the output of the bundle is reshaped into a linear arrangement to form the spectrograph's entrance slit. The star fibers lie at the center of the slit while the sky samplers are located some distance apart to avoid any crosstalk between the desired signal and its related noise. Three "dead" fibers separate the first sky fiber from the star ones to carry out this task. No light is transmitted by these elements. According to Kitchin's nomenclature, this multi-strand cable may be qualified as "coherent" since there exists a logical reshape between the input and output faces of the bundle ${ }^{10}$.

Eventually, the total length of the bundle is $15 \mathrm{~m}$ to ensure a comfortable connection of the telescope to the spectrograph from its separate enclosure whatever movement the observer performs. Special gluing was used by the manufacturer to limit the stresses in the bundle and the FRD they would induce. A large cladding of $125 \mu \mathrm{m}$ is advised by the manufacturer for better stability. The core material is made of pure fused silica and is optimized for high transmission in the near-infrared. Eventually, a stainless steel protection tube surrounds the bundle in order to prevent any unwanted damage that may be disastrous for throughput efficiency.
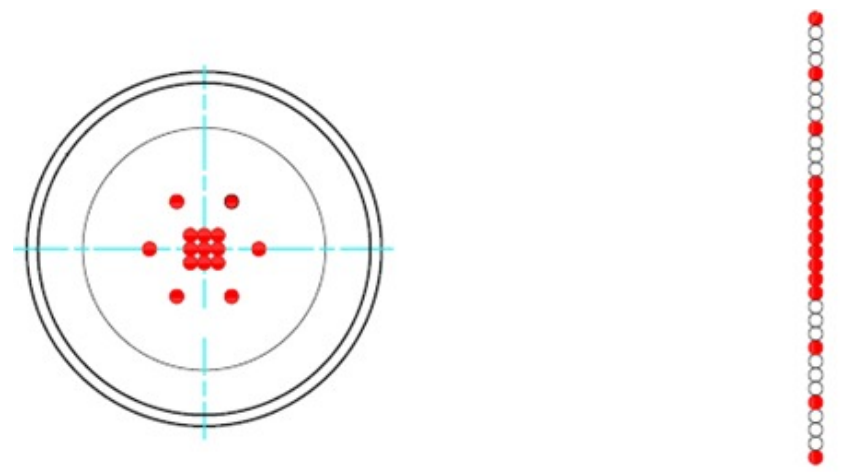

Figure 1. Fiber bundle configuration: input at telescope (left) and output to spectrograph (right)

\section{ALIGNMENT AND OPICAL PERFORMANCE VERIFICATION}

\subsection{Alignment strategy}

To align the spectrograph instrument, a fiber illumination system was first implemented (see Figure 2). This set-up consists in two laser sources that are coupled to the fiber bundle that will be used at the TIGRE telescope. The first light source is a visible HeNe laser which emits at $632.8 \mathrm{~nm}$ while the second one is a Nd:YAG whose emitted wavelength is equal to $1064 \mathrm{~nm}$. Those light sources are coupled with a dichroic window which bends the laser beams to a spatial filter made of two microscope objectives that are separated by a pinhole. Two polarizers can then be rotated with respect to each other to set the illumination level to a proper value and avoid detector saturation. Eventually, another microscope objective focuses the light into a selected fiber within the bundle.

This fiber illumination system features both visible and near-infrared options. This versatility is a very handy tool as it enables to first align the spectrograph in visible light and then proceed with a near-infrared wavelength that is located close to the central scientific wavelength of $1050 \mathrm{~nm}$. Indeed, the practical alignment was first performed in visible wavelength since only one chromatic element is contained within the spectrograph. When switching to the near-infrared waveband, only a focus correction is required to recover the best alignment position. 


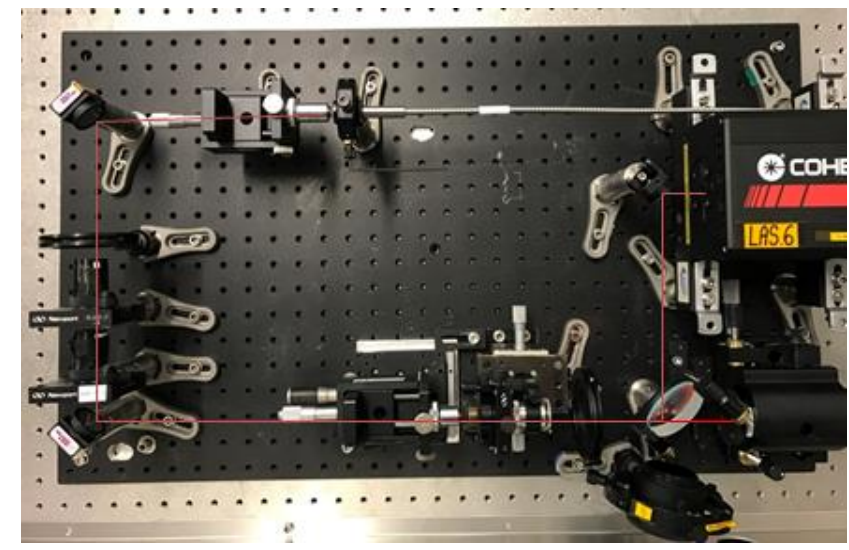

Figure 2. Fiber illuminating system consisting of two co-aligned lasers, respectively for visible (HeNe at $632.8 \mathrm{~nm})$ and near-infrared (Nd:YAG at $1064 \mathrm{~nm}$ ) alignments.

\subsection{First alignment and optical performance evaluation with monochromatic light}

The visible alignment of the spectrograph was performed with the help of the second diffraction order of the grating. This configuration only required a slight modification of the grating's orientation since the available laser wavelengths approximately differ by a factor 2 . Every surface was positioned at its theoretical location and then tuned with the help dedicated micrometer setting screws on the associated optical mount. Once every surface of the spectrograph main channel was correctly installed, the overall optical quality was evaluated by feeding the system with near-infrared light this time.

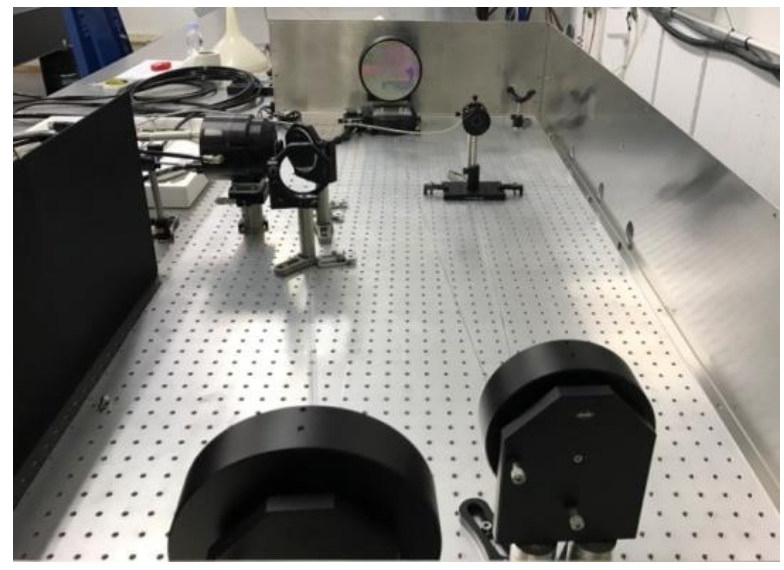

Figure 3. Full spectrograph main channel assembly: the diffraction grating is located at the rear while the collimating and focusing mirrors are visible at the bottom of the image. The toroidal lens and the detector are visible on the left-hand side of the image.

Figure 4 introduces both simulated and observed bundle images. The experimental picture of the bundle comes from the superposition of the individual fiber images since only a single fiber can be illuminated at once with the microscope objective from the fiber illumination system. The whole bundle may be irradiated with the help of a diffuser located between the fibers and the microscope objective but this is avoided as it leads to non-uniform illumination of the fibers and poor spot size evaluation. A speckle pattern arises from the interference of the several modes running through the selected multimode fiber and superposes to the fiber images. Due to this interference phenomenon, the fibers look like being laterally shifted from each other but they are not as will be confirmed with polychromatic light further in this section. The individual pictures were obtained by averaging several frames while scrambling the bundle but the effect remained visible. The simulated average RMS spot size of $35 \mu \mathrm{m}$ is closely approached since the experimentally measured one amounts to $45 \mu \mathrm{m}$. This monochromatic evaluation must then be extended to the whole spectral waveband of interest to assess the resolving power not only at the central wavelength. 

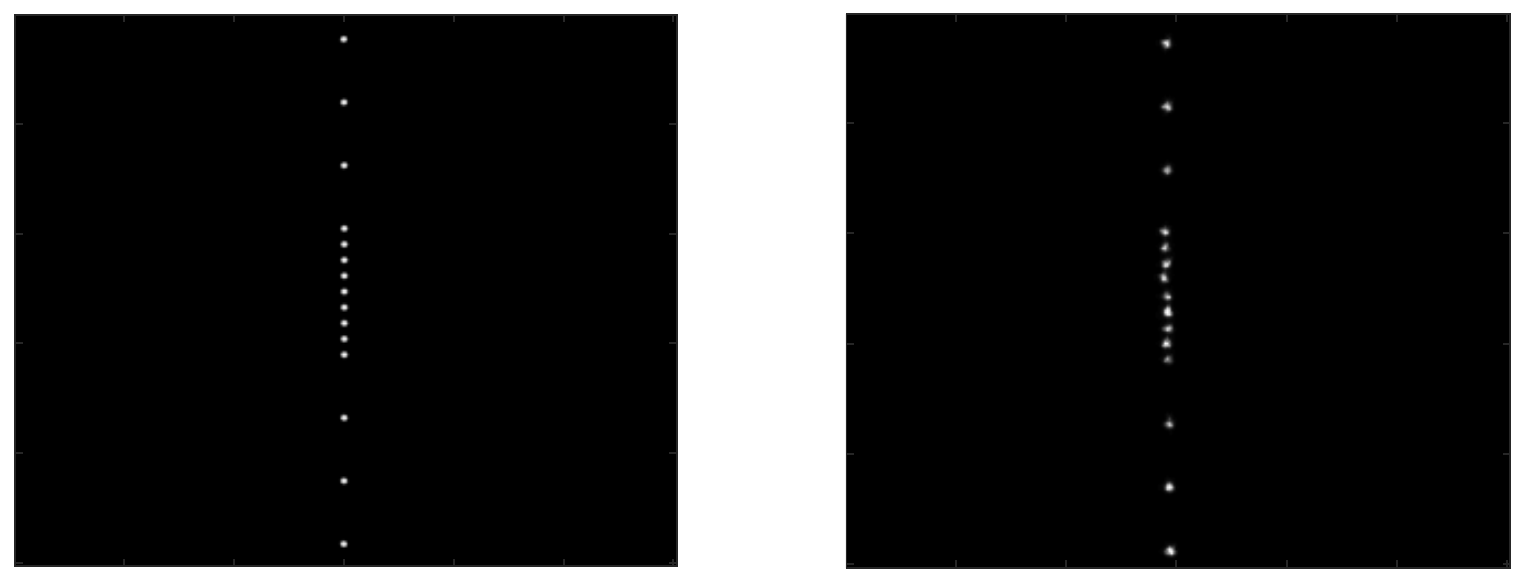

Figure 4. Simulated (left) and measured (right) bundle images.

\subsection{Polychromatic performances}

The previous section illustrated the different steps the visible and near-infrared alignments were made of. The optical performances of the instrument were evaluated with monochromatic laser light in order to measure the Point Spread Function of the spectrograph. This section now introduces the polychromatic behavior of the instrument. For that purpose, the fiber injection system presented in Figure 2 was modified in order to enable the fiber bundle to be lit with polychromatic light. Indeed, the polychromatic performances of the instrument were investigated by observing UNe spectra generated with the help of a hollow cathode lamp from Photron ${ }^{1}$. This way, the resolving power of the spectrograph can be measured by first calibrating the dispersion relation through the focal plane and then evaluating the spectral profile of selected lines. The identification of the observed spectral lines is performed by comparing the experimental spectrum with the atlas from Redman et al. ${ }^{11}$.

Spectra were recorded at respectively central wavelengths of $945.5 \mathrm{~nm}, 994.9162 \mathrm{~nm}, 1006.1 \mathrm{~nm}, 1059.30 \mathrm{~nm}, 1071.9$ $\mathrm{nm}$ and $1119.03 \mathrm{~nm}$ (see Figure 5 and Figure 6). These wavelengths span the whole scientific waveband of interest and investigating the spectrum quality at these locations enables exploring the instrument's global optical efficiency. On the other hand, these precise regions of the spectrum were selected for the existence of specific doublets. These latter were identified by a dedicated algorithm looking for close lines that can be distinguished only if the spectrometer exhibits a high enough resolving power. The distinguishability of these lines therefore enabled a quick visible qualitative assessment of the instrument performances.

Figure 5 (a) and Figure 6 (a) and (c) contain at least one Ne line as can be observed from the reduced spectrum which exhibits at least one pronounced intensity peak. This remarkable feature is easily noticed as it completely outshines all uranium lines. These may be used during spectral calibration to quickly recognize some spectral features and identify subsequent uranium lines. Then, the dispersion relation calculation should avoid using those lines as they suffer from several perturbation effects and are less stable. When a Ne line appears in the spectrum of Figure 5 and Figure 6, these are intentionally truncated in order to emphasize fainter $\mathrm{U}$ lines.

\footnotetext{
${ }^{1}$ A cautious choice of the hollow cathode lamp is important. Indeed, two lamps were tested within the experimental setup. The first one was the Superlamp model, which we selected because it was presented as more luminous than the standard version. A major issue with such a lamp is that only the primary line of uranium is visible whereas all secondary lines disappear, making such lamps unsuitable for spectral calibration.
} 

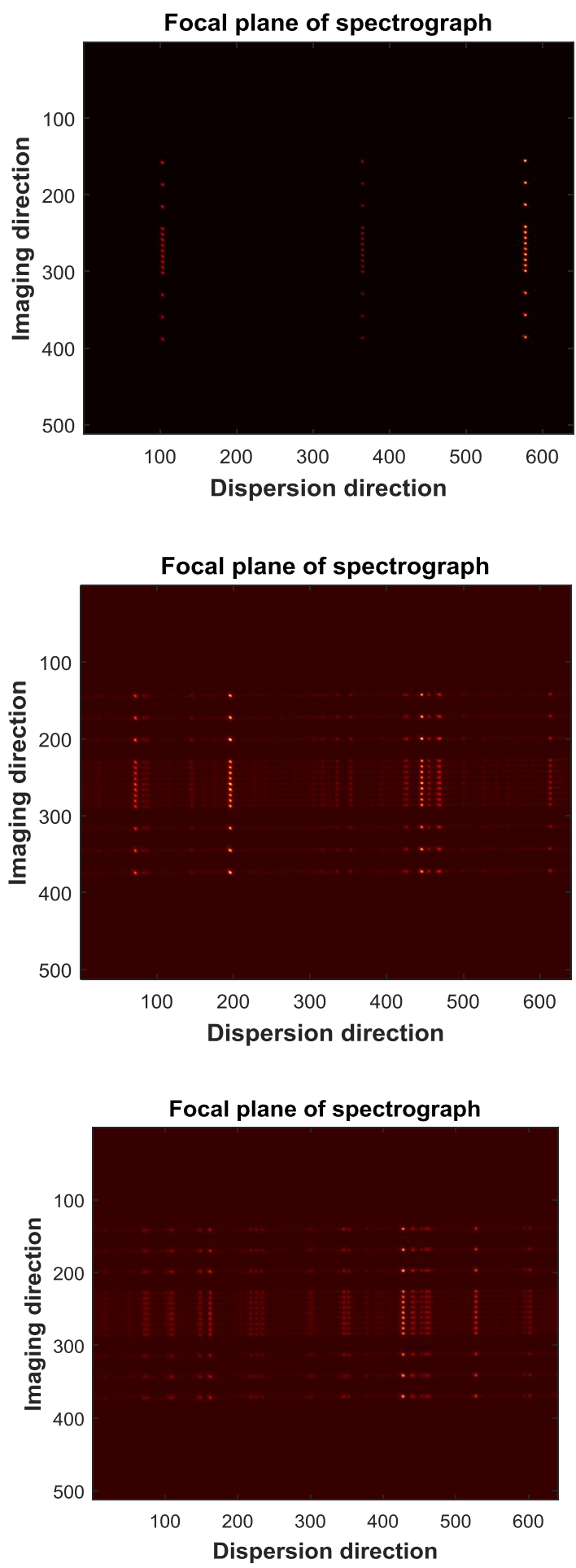

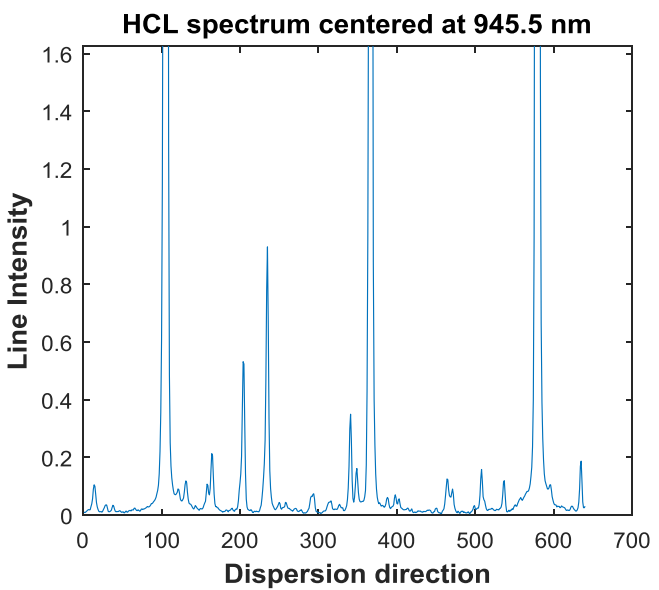

(a)

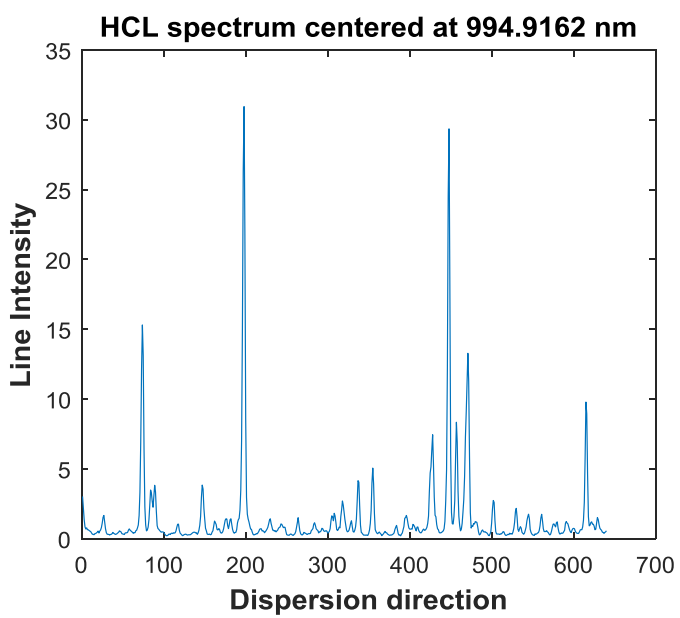

(b)

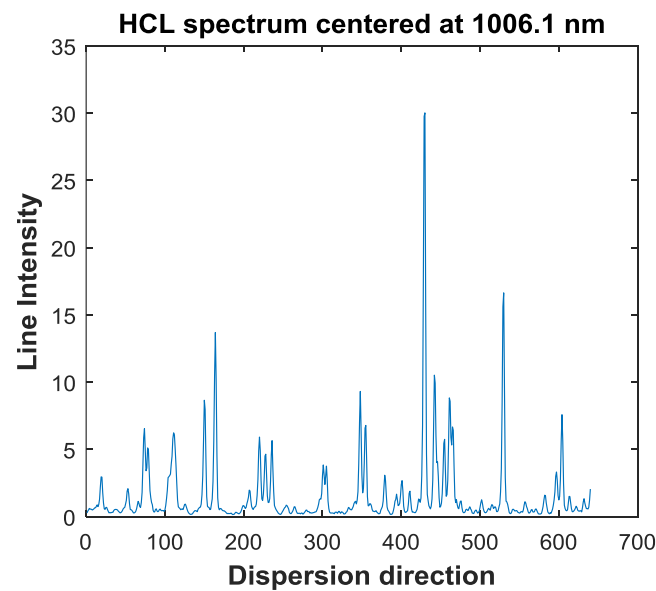

(c)

Figure 5. Focal plane images (left) and reduced spectra (right) when observing the HCL spectrum centered at (a) $945.5 \mathrm{~nm}$ (b) $994.9162 \mathrm{~nm}$ and (c) $1006.1 \mathrm{~nm}$. 

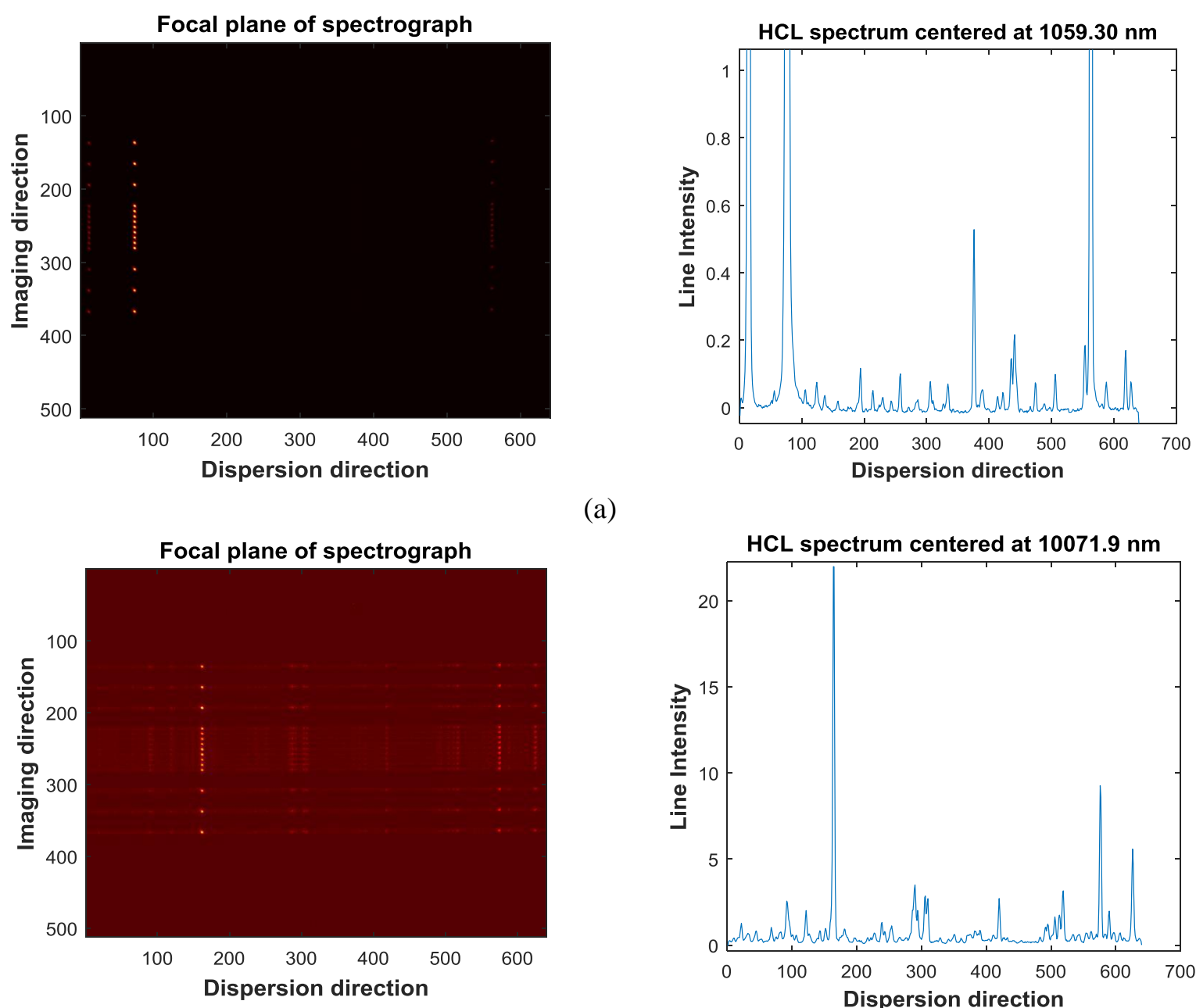

(a)

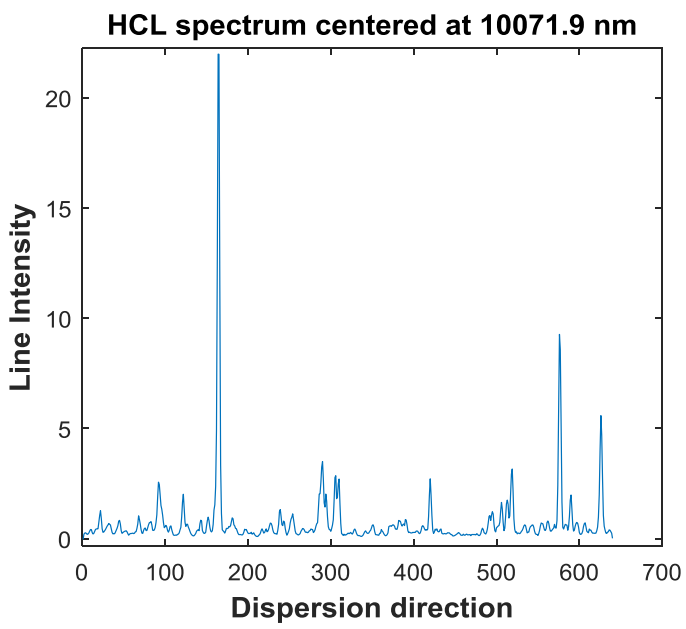

(b)
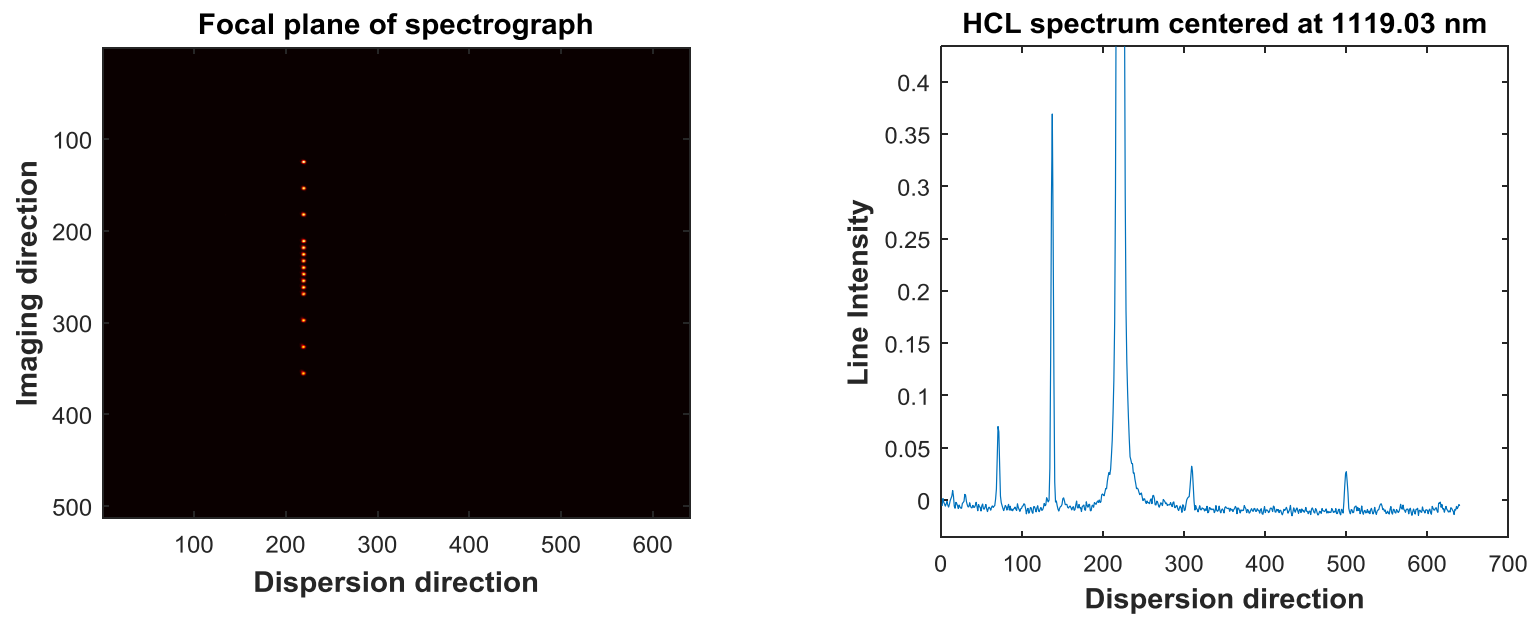

(c)

Figure 6. Focal plane images (left) and reduced spectra (right) when observing the HCL spectrum centered at (a) 1059.30 $\mathrm{nm}$, (b) $1071.9 \mathrm{~nm}$ and (c) $1119.03 \mathrm{~nm}$. 
A quantitative evaluation of the resolving power was the carried out. Figure 7 illustrates the reduced spectrum previously shown when observing the UNe HCL and centering the rotation stage at $1050.30 \mathrm{~nm}$. Redman's atlas was then used to identify several lines an derive the dispersion relation through the focal plane with the help of a polynomial fitting. Both Redman's atlas and the calibrated spectrum are superposed in Figure 7 to illustrate the fine matching between each other. Both spectral positions and intensities are recovered finely except for the $\mathrm{Ne}$ line intensities for reasons previously introduced.

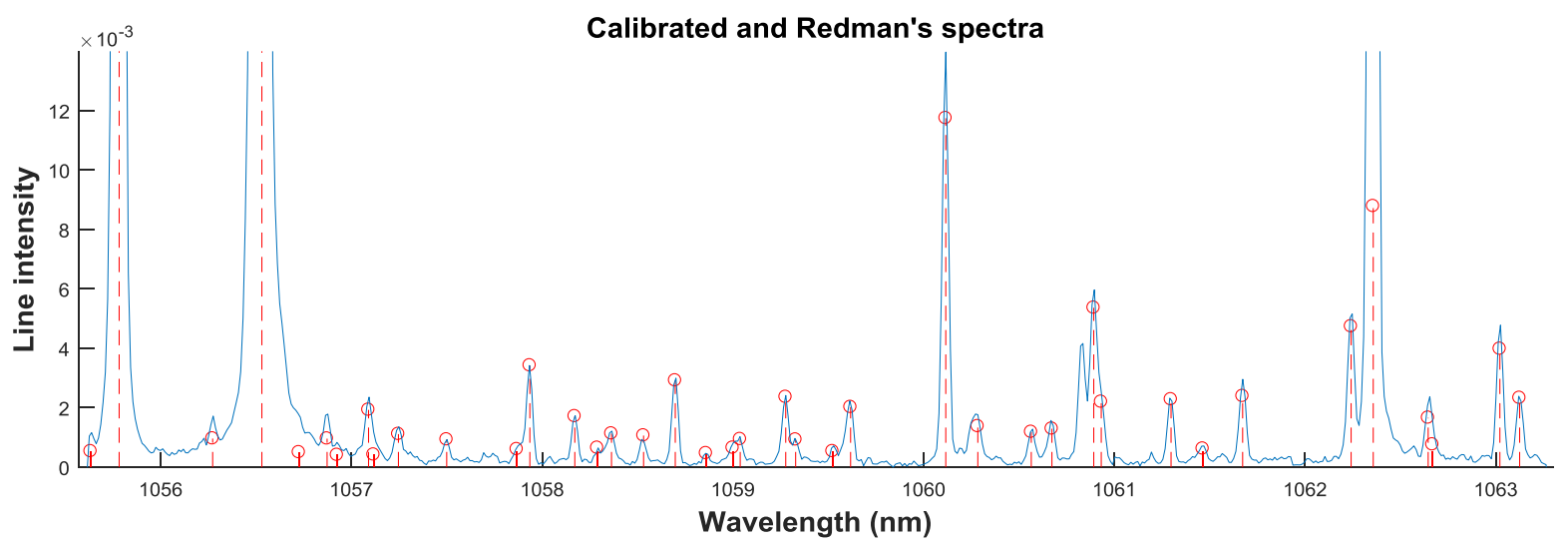

Figure 7. Redman's and calibrated recorded spectra superposed on top of each other.

Then, the resolving power of the instrument was measured on several selected spectral lines. For example, the brightest available $\mathrm{U}$ line is chosen in this case, which is the leftmost on the above spectrum in Figure 7 (this one was deliberately truncated due to its high intensity). On the other hand, this line is isolated from any neighboring spectral line and its spectral profile can be easily computed. The FWHM obtained from this spectral line enables then the calculation of the resolving power. This process is presented in Figure 8.

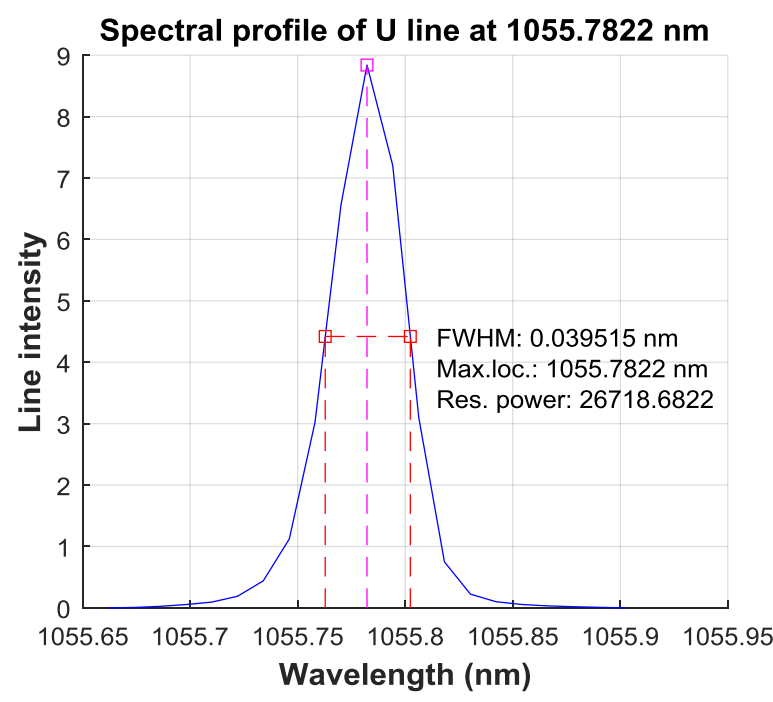

Figure 8. Spectral profile of the line centered at and calculation of the associated resolving power.

The measured FWHM amounts to $0.039 \mathrm{~nm}$ and finely matches the value deduced in the resolving power analysis that was equal to $0.038 \mathrm{~nm}^{6.7}$. The evaluated resolving power amounts to 26718 , which is very close to the value that was deduced from the observation of a pair of very close lines in the UNe spectrum (26000). The test of other lines spread over the spectral band of interest led to the same order of magnitude and the average value of approximately 27000 may be reasonably taken as a sensible baseline for the resolving power of the spectrograph. 


\section{ON SKY OBSERVATIONS FROM LIEGE}

Before shipping the instrument to the TIGRE telescope, tests were performed on the Belgian sky from the Centre Spatial de Liège. These intended to assess the instrument performances by observing bright stars with a small telescope. On the other hand, these tests procured some hands on experience and enabled debugging the spectrograph manipulation before using it on site.

\subsection{Adapted photometric budget}

As a first step, this section intends to investigate whether enough photons could be collected with the selected telescope to obtain useful scientific data. The photometric budget previously performed for the TIGRE telescope was thus adapted to simulate the observation of bright stars with a small telescope, a Celestron NexStar 8 SE. The aperture of this telescope is 8 in and its focal length is equal to $2 \mathrm{~m}$.

The choice of the target for our test observations was guided by several constraints. These included the fact that the telescope had a rather limited aperture and the foreseen difficulties to guide the telescope in such a way that the stellar flux enters the fiber. Therefore, in order to maximize our chances to collect a useful spectrum, we decided to restrict our list of possible targets to the very brightest stars of the Northern autumn sky. Moreover, we wanted to make sure that the star would remain observable over a large fraction of the night. This condition excluded stars with too low a declination, as well as objects which were in the Western half of the sky at the beginning of the night.

The calculated observing times are shown in Figure 9. These amount to a few minutes which should limit the required tracking period of the small telescope.

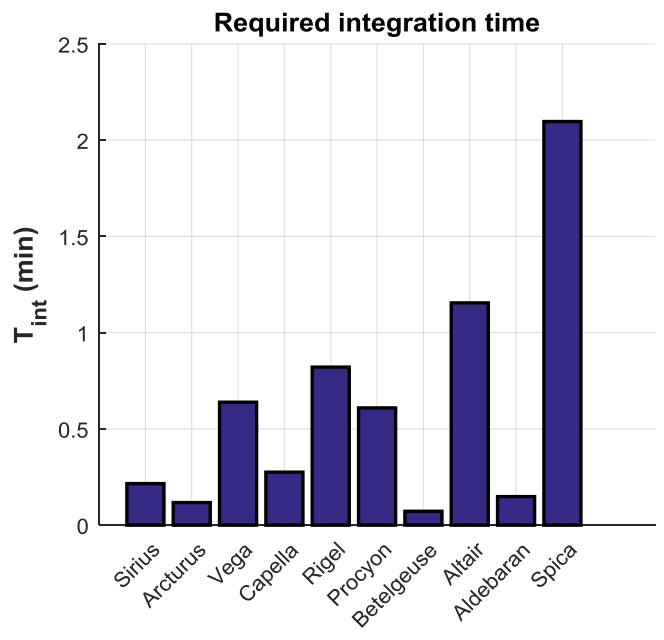

Figure 9. Required integration time at $1050 \mathrm{~nm}$ for the identified targets

\subsection{Preparation of the observation setup}

The photometric budget highlighted the possibility to observe the identified luminous targets with a small telescope located in Liege. This section then intends to introduce the successive practical steps that led to the instrument Belgian first light. Indeed, several technical issues were faced during the first observations and the photon transmission chain from the telescope down to the instrument was established after several iterations.

Once the instrument alignment was completed, blackout material was applied on its internal panels to attenuate reflections of light within the instrument enclosure. These absorbing sheets attenuate the straylight generated by light leakages from the regular light path of the instrument. Figure 10 introduces the instrument final configuration. 


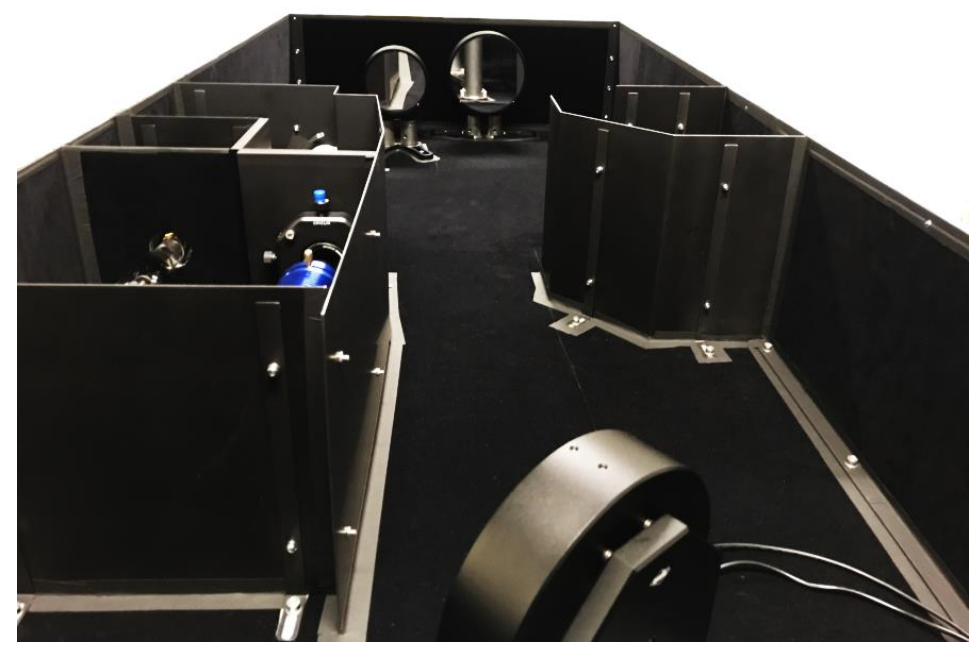

Figure 10. Final configuration of the instrument.

Other complementary equipment was also added to the spectrograph. The fiber bundle and the calibration slits were equipped with mechanical shutters for dark frames recording. These latter are located as far as possible from the detector as advised by Dallier et $\mathrm{al}^{12}$. This way, the entire spectrograph environment is captured when recording dark frames. It may be possible in the future to re-locate the bundle shutter within the SPS for better results. An LED, which intends to back-illuminate the fiber bundle when performing the tracking alignment, was eventually positioned. An achromatic doublet is used to image the LED onto the fibers by reflection through the folding mirror. The control of these shutters and the LED incorporated inside the spectrograph assembly is performed with the help of a dedicated controller. This small programmable electronic platform establishes the connection with the control computer of the spectrograph. The general control unit of the instrument is supplied with a custom software. This latter enables several operations to be carried out such as the calibration or the scientific observation. Both manual and automatic modes were implemented. This enables to either manually control each component separately or run automatic sequences with no required external action.

The spectrograph was then moved from the alignment laboratory to another room suited for night astronomical observations with direct access to the roof. The observation room is depicted in the left panel of Figure 11 where the instrument, its control computer and the fiber illuminating system can be seen. Figure 11 (b) illustrates the removable cover plates which were manufactured within the instrument enclosure. These latter can be rotated in order to provide an easy access to specific optical elements while keeping the instrument cover plate in place. This way, a realignment of the instrument is enabled by finely tuning the position of specific optical elements.

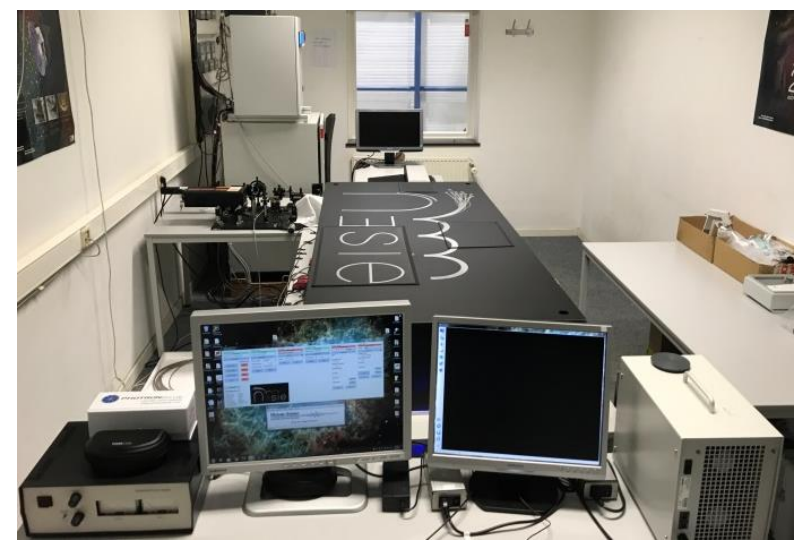

(a)

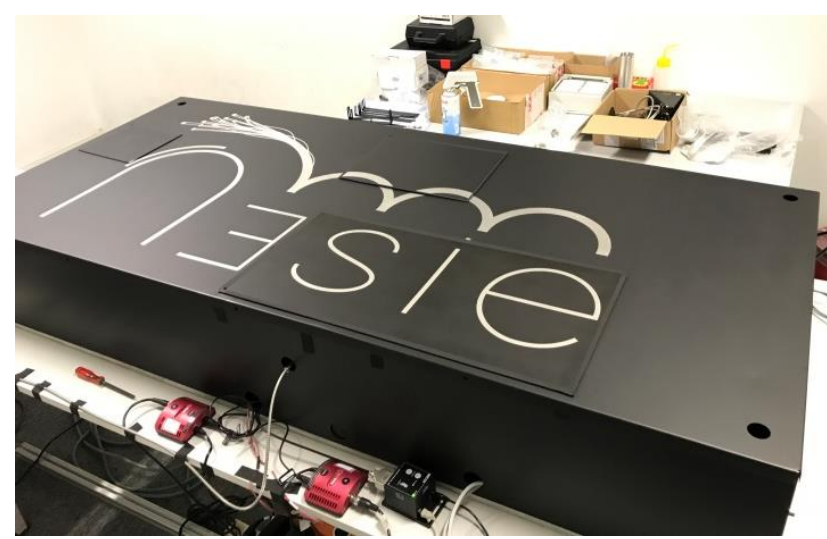

(b)

Figure 11. Observing control room (a) and final instrument configuration with removable cover plates (b). 
On the other hand, a network connection was installed up to the roof in order to be able to remotely drive the spectrograph from a laptop. The operations of the telescope and the instrument are therefore both carried out from the observation site, which largely eases their synchronization. Figure 12 illustrates the observation setup that was installed on top of CSL facilities for the observation trials: the telescope, equipped with the fiber bundle, and the remote laptop.

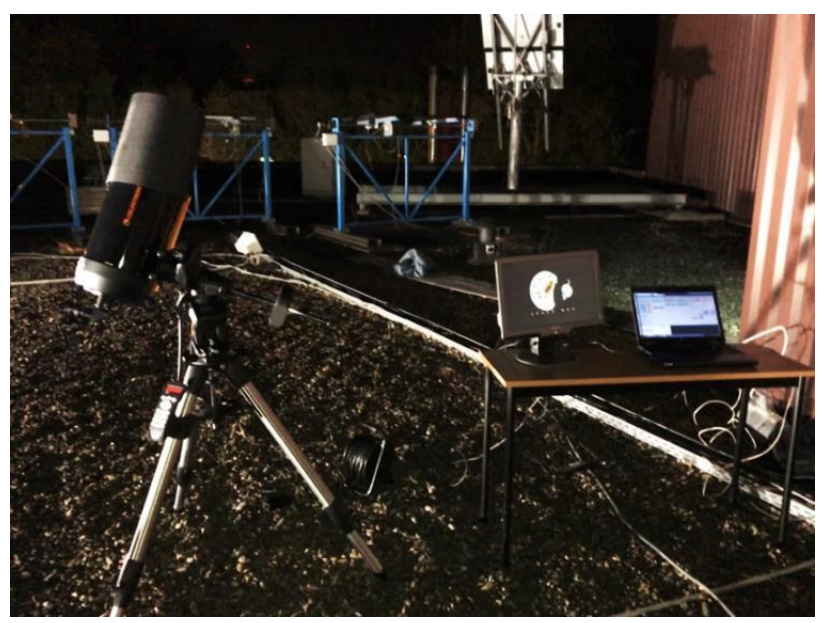

Figure 12. Observation setup installed on CSL facilities roof. The laptop was dedicated to the spectrograph control.

\subsection{Coupling with the telescope and first tests}

The telescope that was used for the observations on the roof of CSL facilities was originally equipped with a flipping mirror accessory located close to its focal plane (see Figure 13 (a)). The initial purpose of this component is to enable pointing at the target star with an eyepiece by reflection with the incorporated mirror and then take an exposure with a camera by flipping this latter out of the field of view. This convenient device was used in a first stage to position the fiber bundle onto the star image and transmit the light to the spectrograph. The bundle mount thus took the place of the camera within the flipping mirror assembly for our purpose. In order to connect the telescope to the spectrograph, an adapted fiber holding system must therefore be designed and manufactured. This latter must account for the telescope optical design and position the fiber at its focal plane while using the flipping mirror device. This custom flange intended to couple the fiber bundle to the flipping mechanism through its translation mount. Once assembled, the overall system enables finely tuning the focus position of the bundle while also scanning the telescope focal plane and center the stellar image.

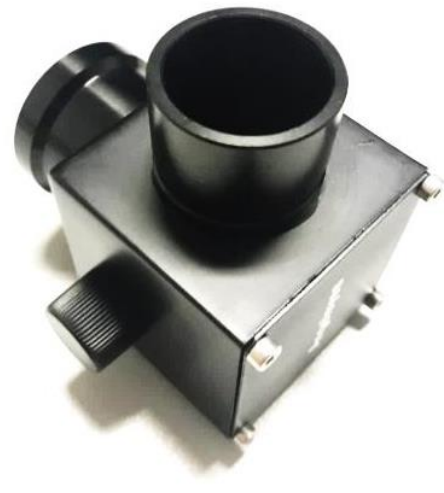

(a)

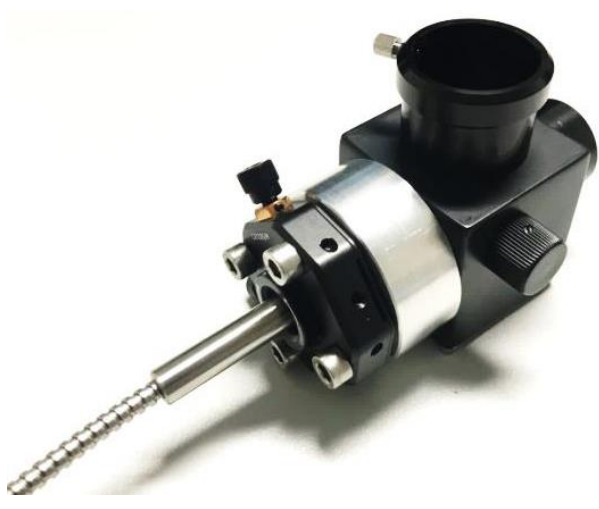

(b)

Figure 13. Flipping mirror device: the upper flange connects to the telescope, the left connecting tube intends to hold an eyepiece and the lower hidden aperture incorporates a standard thread for cameras. The small turning button activates the flipping mechanism (a). Fiber bundle connected to flipping device with translation mount and custom flange. 
First observations were conducted with this setup on June $22^{\text {nd }} 2017$ by pointing Arcturus. As a complement to the telescope guiding, micrometer setting screws onto the fiber optical mount were used to finely align the star image onto a selected fiber. In order to identify the best flux transmission condition, the spectrograph was set at the zeroth order in order to maximize the light visualization as no dispersion occurs in those conditions. The best transmission situation was therefore examined at the spectrograph detector. It must be noticed that doing so does not logically enable both tracking and recording tasks since the grating inclination angle changes from one operation to the other. Several issues appeared and avoided the record of any good-quality result for a few nights. These issues were:

- Fibers appeared to twinkle until the moment they turned off one after the other. The guiding of the telescope was the source of perturbation. A drift in the guiding mechanism actually continuously shifted the star image across the focal plane leading to a variable illumination of the fibers. This explains the fact that fibers were transmitting the photon flux in turn and the difficulty to align the star image onto a fixed selected zone of the bundle. Moreover, a defocus of the Schmidt-Cassegrain telescope induced another artifact: the observed star image pattern exhibited a dark hole in its center. Indeed, when defocusing such a telescope, the secondary mirror obstruction appears and the circular pattern starts looking like a donut. When centering the star image onto the fiber bundle, only the "satellite" fibers were therefore illuminated and this falsely appeared as a lateral misalignment. Refocusing the telescope only made the centering challenge even more dramatic.

- We also noticed the flipping mirror repeatability was not sufficient to ensure the perfect superposition between the star and the fiber. These complications made it difficult to perform good quality observations.

- Moreover, we discovered the stability of the floor to be an issue. The flat roof of CSL facilities is indeed made of a membrane covered by gravels. In addition to the poor telescope-ground contact conferred by the gravels, the soft membrane changed in shape as we were moving on it. Any movement while observing therefore modified the telescope pointing accuracy and the observation suffered from those perturbations.

- Our data reduction pipeline was not optimized. Our primary idea to maximize the signal-to-noise ratio of our images was to acquire multiple frames and stack them. This stacking was originally performed within the detector to limit the amount of data as only the average image was released. Due to the lack of pointing accuracy, many background frames were accounted for within this averaging process, ruining the end-product image.

A few nights were dedicated to try to achieve a proper light transmission down to the spectrograph but no satisfying result was obtained with that strategy. A revised plan was therefore required to achieve the first light and obtain a decent spectrum. Indeed, without a direct view at the scene in the bundle plane, obtaining proper scientific observations was extremely difficult. The conditions were not suitable neither to establish nor to maintain a proper light transmission chain and record the stellar spectrum. In order to overcome the tracking issues we were facing, we developed a better-suited star positioning system. Our previous tests clearly pointed out the need of having a direct view onto the bundle surface. This way, we would be able to observe the actual star image position and superpose it onto a selected fiber. To identify the fiber position, we could benefit from the available lightning solution that was already incorporated inside the instrument. Indeed, the designed star positioning system that will be used at TIGRE requires the bundle to be retroilluminated with the help of a LED ${ }^{6.7}$. This LED could therefore be used for the same purpose: observing the fiber onto a camera positioned at the telescope side of the bundle. The adapted visualization system could therefore mimic the methodology of the star positioning system designed for TIGRE.

The required modification to be applied on our system was therefore to incorporate a small camera and replace the flipping mirror by a beamsplitter. Indeed, both the star image and the fibers must be observed simultaneously. The technique that was considered is not exactly the same as the one employed for the real star positioning system. As a reminder, this latter incorporates an antireflective window, namely the beamsplitting element, which selects the light both from the telescope and the fibers. To do so, fibers are projected onto a SBIG camera with the help of a small mirror. In the present case, for simplicity, compactness reasons and availability of the equipment, another strategy was adopted. The camera featured an objective which focused directly onto the bundle plane. This way, the fiber locations could be identified when the fibers were lit from the spectrograph. On the other hand, since the bundle end is planar and metallic, the induced reflectivity could be used as a mirror to visualize the star by reflection as long as it remained away from a 
fiber. As soon as the star flux was transmitted through a selected fiber, the image disappeared from the camera focal plane and the transmission occured. The optical design of the modified tracking device is depicted in Figure 14.

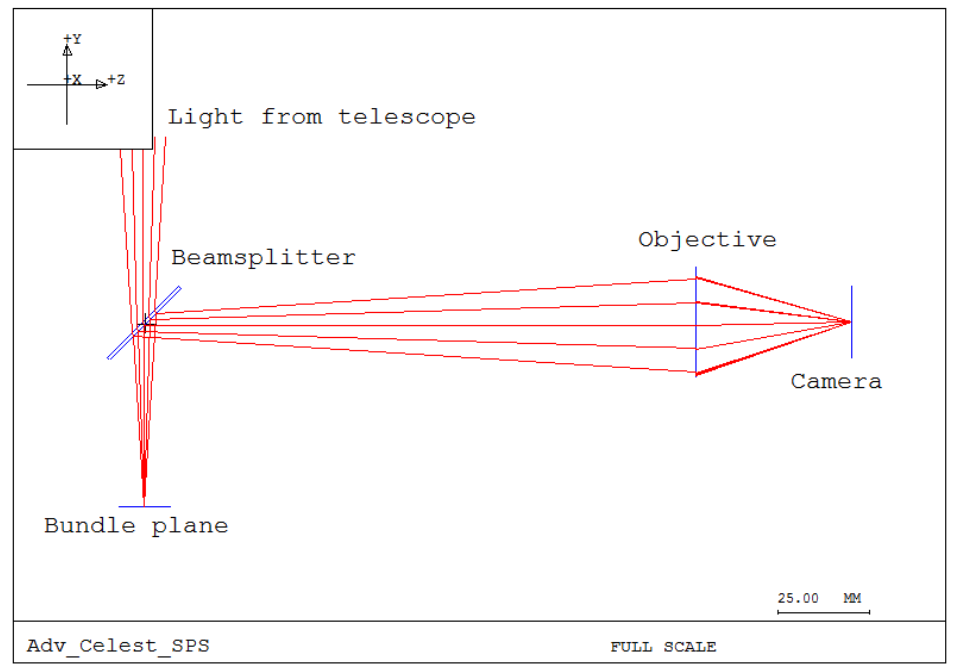

Figure 14. Improved tracking device optical design. Light propagates from the telescope down to the bundle plane. The reflected beam onto the metallic ferrule (when not centered onto a fiber) then goes back to the beamsplitter along with the light from the fibers and reaches the camera.

The camera we used for the visible alignment of the spectrograph was chosen to integrate the new tracking instrument. Both its interface and compactness eased its integration. In order to insert the beamsplitter within the flipping mirror containing box, the mechanism of this latter was replaced by a threaded shaft holding a U-shaped metallic mount. This latter intended to support the beamsplitter which replaced the flipping mirror. Teflon plates were used to sustain the beamsplitter within this custom optical mount. The beamsplitter mount and its modified assembly are presented in Figure 15 (a). In order to position the camera the correct distance away from the fiber bundle, a circular tube with setting grooves was manufactured. This latter enabled the camera piston to be adjusted to finely focus onto the bundle and clearly distinguish the different fibers. The obtained visualizing apparatus is depicted in Figure 15 (b).

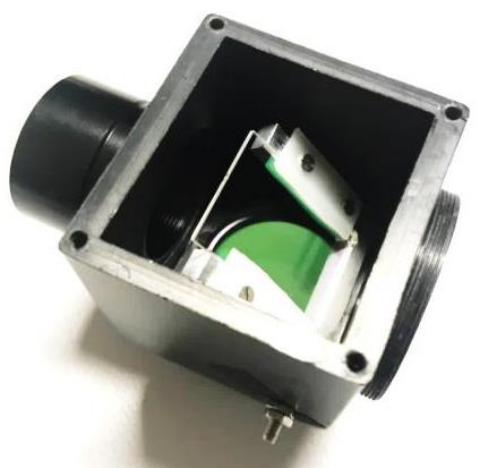

(a)

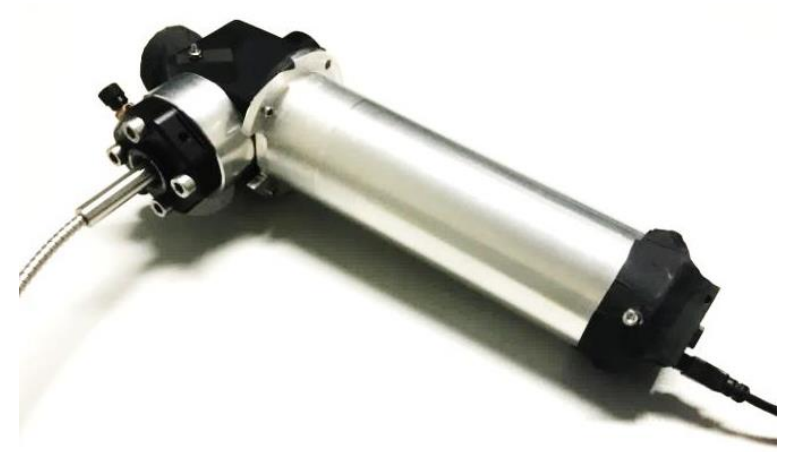

(b)

Figure 15. Modified flipping mirror box (a) and adapted tracking device (b).

The next observing sessions were conducted with the help of the updated tracking system. This adapted system enabled a real live tracking of the target and a simultaneous recording of the spectrum. The previous device did not allow both operations to be carried out at the same time since the star alignment was performed using the grating zeroth order. Therefore, switching between the tracking and recording activities required the spectrograph configuration to be modified. Due to guiding uncertainties, the alignment condition had deteriorated while modifying the setting configuration and the recorded frames were therefore of poor quality. The advanced tracking system enabled to first 
follow the target position within the bundle plane and secondly correct for any pointing drift in real time. The added display was thus dedicated to following the target position with respect to the fiber while the laptop was devoted to the spectrograph control.

These observing nights intended to test this update of the tracking device by observing Capella. Indeed, because of its brightness in the near-IR, Capella ( $\alpha$ Aur $=$ HD 34029, $\mathrm{J}=-1.29, \mathrm{RA}=05 \mathrm{~h} 16 \mathrm{~min} 41 \mathrm{sec}, \mathrm{DEC}=+45^{\circ} 59^{\prime} 53^{\prime \prime}$ ) turned out to be the best candidate to receive a maximum of photons. Visualizing both the fibers and the star image was a success. Figure 16 is a live tracking acquisition showing the star image which lies close to the fiber bundle. Visualizing both the target and the bundle occurs when the stellar flux impacts on the metallic ferrule and is reflected to the camera. The fiber bundle is illuminated to be able to locate and select an acquisition fiber. As a second step, a green cross mark is positioned onto this fiber and the bundle turned off. The telescope pointing is then adapted to bring the target onto the cross mark and maximize the photon transmission to the spectrograph. When superposed to the fiber core, the stellar image disappears from the acquisition screen. The goal is then to correct the telescope tracking as soon as the star becomes visible again to avoid light leakages since the spectrograph camera is acquiring data in a continuous way.

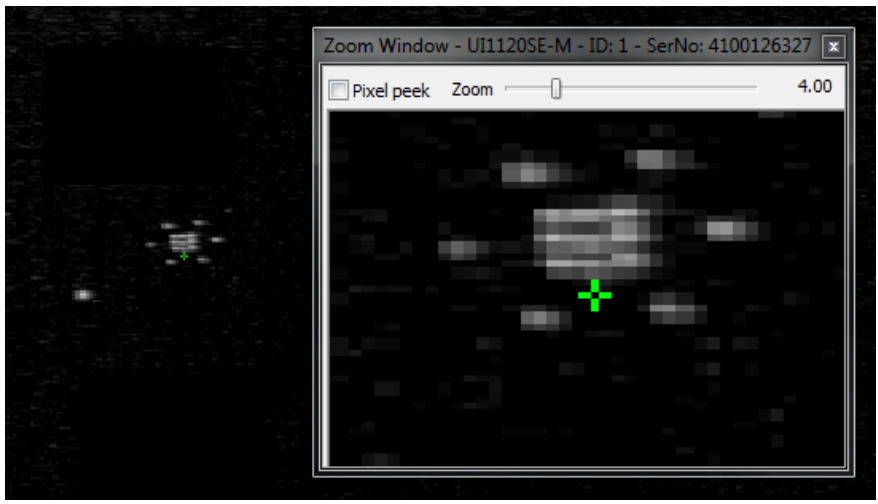

Figure 16. Live tracking acquisition. The star image lies at the left side of the picture and the fiber bundle right above. A zoom onto the bundle enabled to tick a mark onto a fiber to lock its position after having turned off the LED.

This improvement of the tracking device eased the superposition of the stellar image and the fiber bundle. The continuous correction of the telescope guiding however remained a difficult task but more acquisitions incorporated scientific signal.

After having reduced the data from the previous nights, we identified the best recorded frames and their associated conditions. Indeed, the first nights, even though the received stellar flux was low, enabled to test different observing schemes. Several integration times and acquisition options were therefore assessed and the best observing strategy was established as the nights succeeded. For example, we investigated the most appropriate integration time and observed that $15 \mathrm{sec}$ appeared to be the best value. The camera must indeed integrate several seconds to catch enough light to enable the detection. On the other hand, the star image did not lie onto the fiber core more than a few seconds before a readjustment of the telescope was required. Integrating during a long period would therefore imply many intervals when the telescope is not well pointed and no light enters the spectrograph. On the other hand, the observing time was also limited by the high dark signal of the InGaAs detector we selected.

The camera settings were also optimized. We used the Medium Gain to enable integrations of a few seconds while not saturating the detector. As previously mentioned, we also tried during the first nights to average many frames within the hardware as it is enabled by the camera. This way, a limited amount of data is produced per observation as only one light image file is generated: the mean image of the programmed frame sequence. This technique first appeared as a clever method for both limiting the amount of data to process and increase the signal-to-noise ratio of the acquired image. However, due to the limited telescope pointing accuracy, many dark sessions were involved in this averaging process which totally ruined the observations. This approach is closely related to the above conclusion on the upper limit on integration time. A preferred approach was to take a large number of similar frames and record all of them. This way, the selection of the best ones, i.e. those which contain scientific information, was enabled during the data reduction. Only the camera integrations that occurred during a proper synchronization with the telescope guiding were therefore kept for the calculation of the star spectrum. 
The amount of recorded images where the stellar flux was detected largely increased in comparison to the previous observations after this update of the improved tracking device. Moreover, the intensity of the signal surpassed all previous measurements. The combination of data acquired from both nights enabled to largely improve the quality of the reduced spectrum and confirm the instrument's first light on 16 October 2017. The reduced image after combining the observations from both nights is depicted in Figure 17. The illumination of a fiber located close to the $350^{\text {th }}$ line of the detector is clearly noticeable. Several absorption features appear to the naked eye when scanning the spectrum from one side to the other. They appear as dark intervals within the detector band illuminated by the target spectrum.

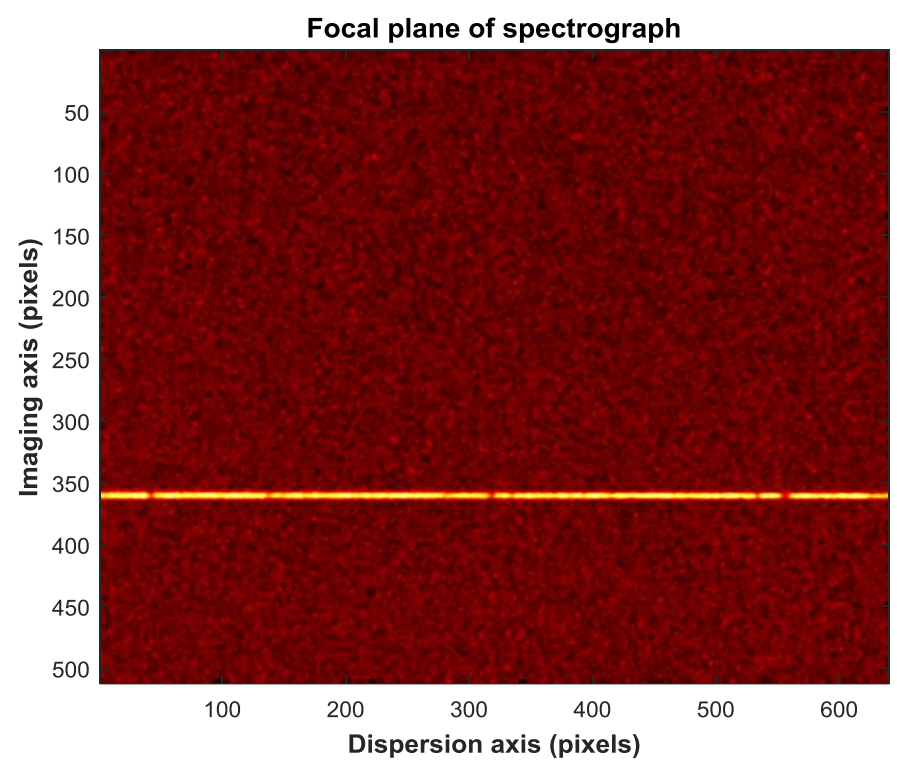

Figure 17. Focal plane image when pointing at Capella with the second update version of the advanced tracking device.

The reduced spectrum from Figure 17 is illustrated in Figure 18. The absorption features appear as sharp decrease in the recorded light intensity. The amount of noise is significantly decreased in comparison with the previously obtained results thanks to the live tracking ability.

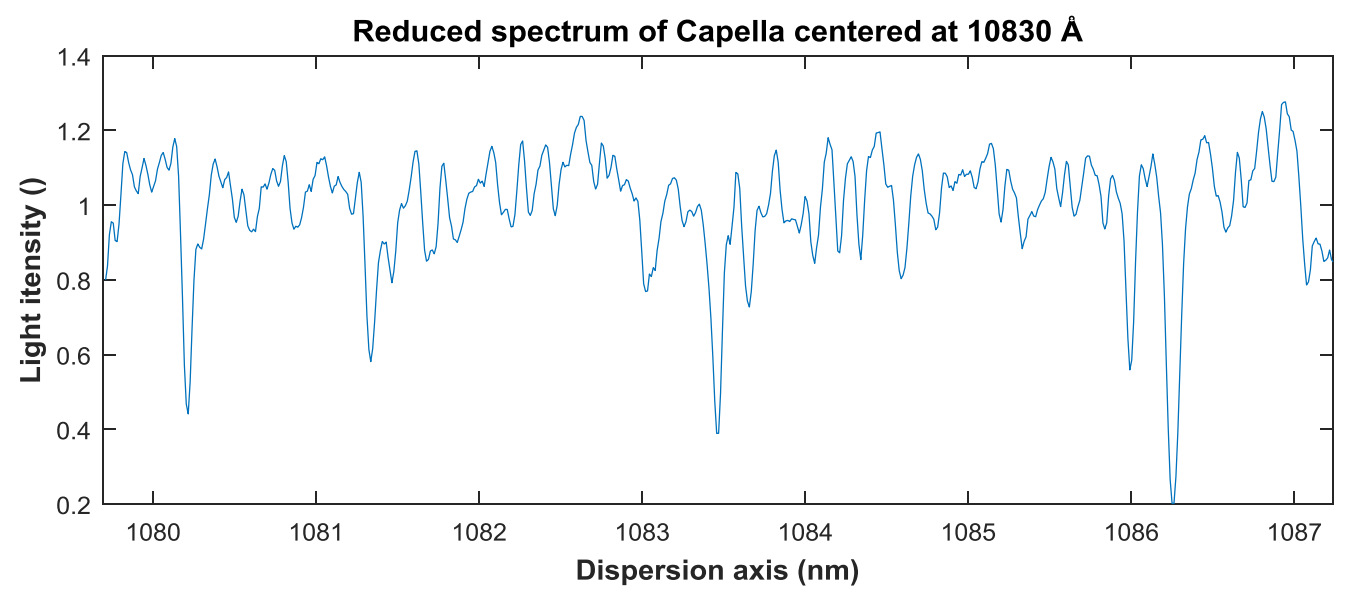

Figure 18. Reduced spectrum of Capella centered at $10830 \AA$ after combining the different observations from the last two nights.

It may appear contradictory that several $15 \mathrm{sec}$ acquisitions were required to obtain a satisfying result. Indeed, the previous photometric budget roughly indicated a similar integration time to reach a signal-to-noise ratio equal to 100 However, the pointing accuracy and the associated corrections led to acquisitions during which the star light was not 
continuously transmitted to the spectrograph. Therefore, dark integrations were mixed to signal recording and the photometric budget did not foresee such complications.

On the other hand, a close look at Figure 16 reveals that the star image is larger than the one considered during the photometric analysis. Indeed, the stellar image onto the bundle plane is poorer than the illuminated fiber core. This would require a typical seeing of 5 arcsec if the fiber bundle is located at the best telescope focus position. This may be another source of image blurring even if we practically optimized the telescope focus by visually minimizing the stellar image onto the camera detector.

Atmospheric turbulence was also probably important because of the strong winds occurring during those nights. Moreover, atmospheric diffraction was probably high as a result of the large airmass during our observations. It might also be noticed that the diffraction limited performances of small telescopes at long wavelengths quickly degrade. Indeed, this small telescope when observing at $1.1 \mu \mathrm{m}$ exhibits a PSF (Point Spread Function) of approximately $30 \mu \mathrm{m}$. This contributor to light losses might therefore have been slightly underestimated too. Whatever the reason explaining an increased star image diameter, the consequence is a longer integration time to reach the same signal-to-noise ratio. Finally we note that the stellar image was disappearing from the tracking screen when it reached the fiber cladding, not the fiber core in reality. In that condition, light is not transmitted and the fine alignment is not complete.

\subsection{Verification of performances}

By the time of our observations (15 and 16 October 2017), Capella was still relatively low on the Eastern horizon at the beginning of the night and was then progressively rising to its near zenith position. Since we performed our observations during the first halves of the two nights, the airmass of the target was relatively high. On the first night, the first and last spectra were taken at airmasses of 3.06 and 1.5, respectively. For the second night, the corresponding range of airmasses was between 3.64 and 1.43. Therefore, one expects the presence of relatively strong telluric features in the spectrum (see below).

Capella is a so-called RS CVn binary system with an orbital period of 104.02 days ${ }^{13}$ (and references therein). RS CVn binaries consist of two late-type subgiants or giants, with (at least) one of them displaying a strong magnetic activity. The magnetically active stars display giant stellar spots which modulate the light curve of these objects as they rotate around their axis and around their common center of gravity ${ }^{14,15}$. Moreover, large magnetic loops may connect spots on both components of the binary system, hence leading to complex field entangling and producing a strong X-ray emission with frequent and substantial flaring ${ }^{16}$.

Mekkaden (1985) reported the detection of a strong dependency between the X-ray luminosity of long-period RS CVn stars and the strength of their He I $\lambda 10830$ absorption line ${ }^{17}$. Therefore, this kind of objects could be scientifically rewarding targets for NGSIE, and we have chosen a central wavelength of $10830 \AA$ for our observation. Nevertheless, let us remind here that the goal of the present observations is not to perform any scientific study, but rather to test the performances of our instrument.

\subsection{The spectrum of Capella around the He I $\lambda 10830$ line}

An important aspect to keep in mind here is the fact that Capella is a spectroscopic binary consisting of a slow rotating (v $\sin \mathrm{i}=4 \mathrm{~km} / \mathrm{s}$ ) G8 III primary and a rapidly rotating (v sin $\mathrm{i}=35 \mathrm{~km} / \mathrm{s})$ G0 III secondary ${ }^{13}$. When interpreting the spectra, we thus need to account for the orbital Doppler shift of the two stars. Our NGSIE observations of Capella were taken between JD2 458042.321 and JD2 458042.426 (first night), and JD2 458043.305 and JD2 458043.439 (second night). According to the ephemerides of Torres et al. ${ }^{13}$, these dates correspond to orbital phases $0.122-0.133$. For the primary star, the expected radial velocity (RV) is $52.8 \pm 0.5 \mathrm{~km} / \mathrm{s}$ where the quoted uncertainty corresponds to half the RV range covered between phases 0.122 and 0.133 . The corresponding value for the secondary star is $6.4 \pm 0.5 \mathrm{~km} / \mathrm{s}$.

Figure 19 illustrates a comparison between our NGSIE spectrum of Capella and the 'summer' atmospheric transmission spectrum of Hinkle et al. ${ }^{18}$. We clearly see that the strongest absorption features in the spectrum (near 10802, 10813, $10835,10837,10860$ and $10862 \AA$ ) are due to telluric lines. A good understanding of these lines is important since the He $\lambda 10830$ line is one of the main drivers behind the NGSIE spectrograph. A close comparison reveals that the telluric reference spectrum is shifted with respect to the NGSIE spectrum. This shift was corrected before we compared our spectrum with the theoretically expected spectrum of Capella. For this latter exercise, we also had to account for the heliocentric correction of $+23.6 \mathrm{~km} / \mathrm{s}$ of Capella at the time of our observations. 


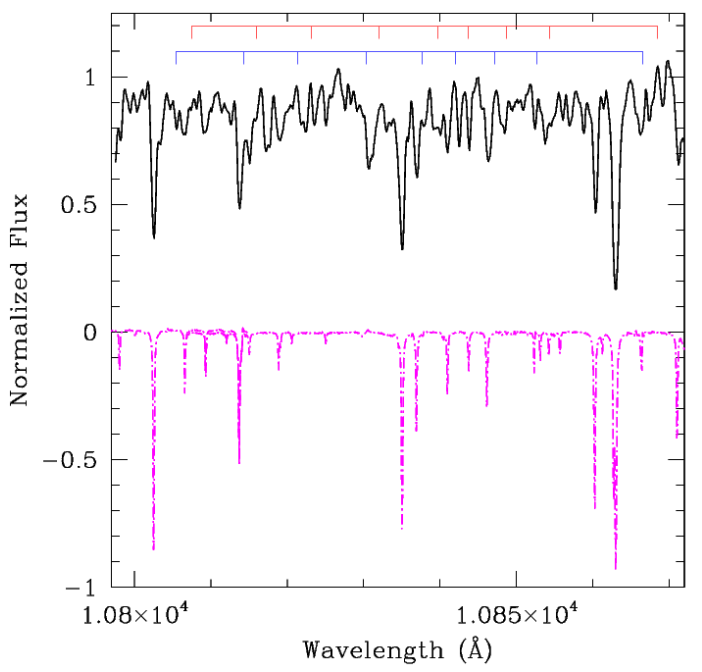

Figure 19. NGSIE spectrum of Capella (black solid line). The magenta spectrum corresponds to the atmospheric transmission tabulated by Hinkle et al. ${ }^{18}$ and shifted for clarity by -1.0 . The red and blue tags indicate the expected wavelengths respectively of the most prominent primary and secondary spectral features.

Moreover, the telluric lines are much broader in the NGSIE spectrum than in the atmospheric transmission spectrum. This is due to the much higher spectral resolving power (100 000) of the atmospheric transmission spectrum ${ }^{18}$. The atmospheric transmission spectrum thus needs to be broadened to match NGSIE's spectral resolution. For the present case, we did not correct the spectrum for atmospheric transmission. Indeed, attempts to do so failed. This is most likely because of the high average airmass and the rather wide range of airmasses during our observations. Such a situation probably emphasizes the non-linear behavior with airmass of the intensity of the telluric lines.

To identify the stellar lines, we have retrieved synthetic spectra computed with the PHOENIX model atmosphere code and tabulated by Husser et al. ${ }^{19}$. To match as closely as possible the actual spectra of the primary and secondary star, we have extracted synthetic spectra with $\mathrm{T}_{\text {eff }}=5000 \mathrm{~K}$ and $\log \mathrm{g}=2.50$ for the primary and $\mathrm{T}_{\text {eff }}=5700 \mathrm{~K}$ and $\log \mathrm{g}=3.00$ for the secondary star. Following the results of Torres et al. ${ }^{13}$, we have adopted solar abundances for both models. These spectra were then shifted in wavelength, according to the orbital solution of Torres et al. ${ }^{13}$, and were broadened to account for the stellar rotational velocity (of the secondary) and the spectral resolution. These synthetic spectra then allowed us to attribute the strongest non-telluric absorptions in the spectrum to the binary components (see Figure 19). In particular, we found a prominent He $\lambda 10830$ line, mostly associated with the active secondary star (see Figure 20).

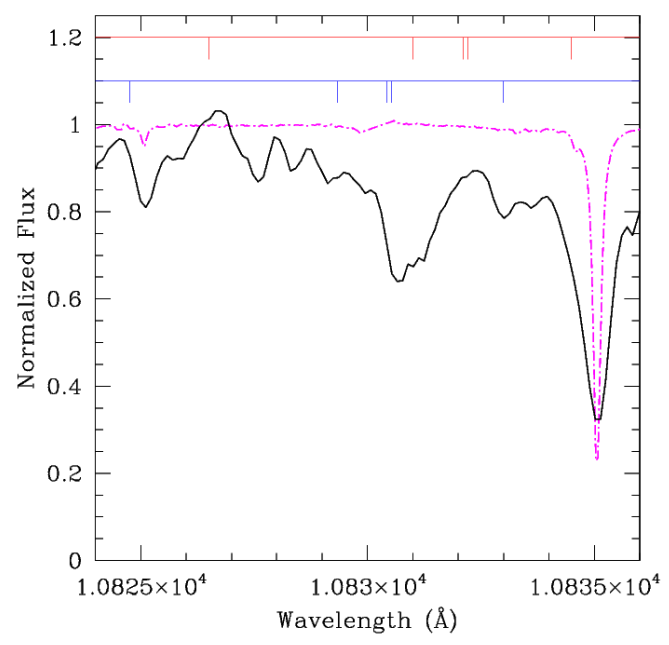

Figure 20. Zoom on the spectral region around the He $\lambda 10830$ triplet. The atmospheric transmission is now overplotted on the NGSIE spectrum. The other symbols are as in Figure 19. 


\section{CONCLUSION}

Our contribution highlighted the aim of our scientific association: increasing the knowledge on massive stars and better understand their mechanisms. For that purpose, three entities form the University of Liege joined their forces to cover both theoretical and technical aspects: GAPHE and ASTA aim at improving their models with the help of observational data provided by new instruments developed at CSL.

The conception and design analyses of NGSIE were presented in a dedicated previous paper and this contribution focuses on the experimental performances ${ }^{6,7}$. The alignment of the instrument was first carried out in visible light and then checked with near-infrared laser radiation. The optical quality and associated resolving power of the spectrograph were eventually assessed by imaging the spectrum of a UNe HCL. The alignment results were in close agreement with the simulations ${ }^{6.7}$ and further tests on the sky followed.

The on-sky observations were performed with a small telescope from CSL installation roof. A photometric budget first assessed the feasibility of this process and the spectrograph was then prepared for observation. The initial equipment was not sufficiently accurate to enable scientific observations and alternate schemes were therefore investigated until the first light was observed on October $17^{\text {th }}$ 2017. The observation Capella, a spectroscopic binary, ended with the derivation of a spectrum that was compared with simulated expectations. A fine matching between both observed and theoretical lines confirmed the ability of the spectrograph to derive fine-detailed spectra even though harsh environmental conditions were faced and a small telescope selected.

These encouraging results foresee a fruitful use of the TIGRE telescope and collaboration between al the ARC contributors. NGSIE is expected to be shipped during the summer of 2019.

\section{ACKNOWLEDGEMENT}

This research was funded through an ARC grant for Concerted Research Actions, financed by the French Community of Belgium (Wallonia-Brussels Federation).

\section{REFERENCES}

[1] Schmitt, J. H. M. M.; Schröder, K.-P.; Rauw, G.; Hempelmann, A.; Mittag, M.; González-Perez, J. N.; Czesla, S.; Wolter, U.; Jack, D.; Eenens, P.; Trinidad, M. A. "TIGRE: A new robotic spectroscopy telescope at Guanajuato," Astronomische Nachrichten 335(8), 787-796 (2014)

[2] Eversberg, T.; Vollmann, K,. [Spectroscopic Instrumentation: Fundamentals and Guidelines for Astronomers], Springer, Berlin (2014).

[3] Massey, P. "Massive Stars in the Local Group: Implications for Stellar Evolution abd Star Formation," Annu. Rev. Astron. Astrophys. 41, 15-56 (2003).

[4] Langer, N. "Presupernova Evolution of Massive Single and Binary Stars," Annu. Rev. Astron. Astrophys. 50, 107-164 (2012).

[5] Zinnecker, H.; Yorke, H. W. "Toward Undertsanding Massive Star Formation," Annu. Rev. Astron. Astrophys. 45, 481-563 (2007).

[6] Kintziger, C., Desselle, R., Loicq, J., Rauw, G., \& Rochus, P., "Conception of a near-IR spectrometer for ground-based observations of massive stars," Proc. SPIE 9908, p. 99085R (2016).

[7] Kintziger, C., Desselle, R., Loicq, J., Rauw, G., \& Rochus, P., "Conception of a near-infrared spectrometer for ground-based observations of massive stars," Journal of Astronomical Telescopes, Instruments, and Systems 3 (1), p. 015002 (2017).

[8] Barden, S. C. "Fiber Optics at Kitt Peak National Observatory," Instrumentation for Ground-Based Optical Astronomy, 250-255 (1988)

[9] Parry, I. R. "Multi-Object Spectroscopy with Optical Fibres," Wide-field spectroscopy 212, 3 (1997)

[10] Kitchin, C. R., [Astrophysical Techniques]," Institute of Physics Publishing, Bristol and Philadelphia (2003)

[11] Redman, S. L.; Lawler, J. E.; Nave, G.; Ramsey, L. W.; Mahadevan, S. "The infrared spectrum of uranium hollow cathode lamps from $850 \mathrm{~nm}$ to $4000 \mathrm{~nm}$ : wavenumbers and line identifications from Fourier transform spectra," The Astrophysical Journal Supplement Series 195(2), 24 (2011)

[12]Dallier, R.; Cuby, J. G. "Noncooled near-infrared spectroscopy," Proc. SPIE 3354, 833-844 (1998) 
[13]Torres, G.; Claret, A.; Pavloski, K.; Dotter, A. "Capella ( $\alpha$ Aurigae) revisited: new binary orbit, physical properties, and evolutionary state," The Astrophysical Journal 807(1), 26 (2015)

[14]Eaton, J. A.; Hall, D. S. "Starspots as the cause of the intrinsic light variations in RS Canum Venaticorum type stars," The Astrophysical Journal 227, 907-922 (1979)

[15] Vogt, S. S.; Penrod, G. D. "Doppler imaging of spotted stars: application of the RS Canum Venaticorum star HR 1099," Publications of the Astronomical Society of the Pacific 95(571), 565 (1983)

[16] Kürster, M.; Schmitt, J. H. M. M. "The longest stellar X-ray flare observed with ROSAT," Astronomy and Astrophysics 311, 211-229 (1996)

[17] Mekkaden, M. V. "The dependence of He I 10830 absorption on X-ray luminosity in RS CVn binaries and very active F and G Main-Sequence stars," Astrophysics and space science 110(2), 413-416 (1985)

[18] Hinkle, K.; Wallace, L.; Livingston, W. "Infrared atlas of the Arcturus spectrum, 0.9-5.3 microns," Publications of the Astronomical Society of the Pacific 107(717), 1042-1046 (1995)

[19] Husser, T. O.; Wende-von Berg, S.; Dreizler, S.; Homeier, D.; Reiners, A.; Barman, T.; Hauschildt, P. H. "A new extensive library of PHOENIX stellar atmospheres and synthetic spectra," Astronomy \& Astrophysics 553, A6 (2013) 